\title{
A FAVOR DE LA NARRATIVA COMO METODOLOGÍA PARA EL ESTUDIO DE LO SOCIAL
}

\author{
IN FAVOR OF NARRATIVE AS A METHODOLOGY FOR THE STUDY OF \\ THE SOCIAL
}

\section{A FAVOR DA NARRATIVA COMO METODOLOGIA PARA O ESTUDO DO SOCIAL}

\author{
Antonio Rueda Mateos \\ Colegio La Salle, España \\ https://orcid.org/0000-0001-8351-5779 \\ antonio.ruedamateos@gmail.com
}

Recibido: 29/07/2020 Revisado:13/08 /2020 Aceptado:14/08/2020 Publicado: 01/11/2020

Resumen: El artículo aquí presentado tiene el propósito de describir y dar a conocer el modo de proceder ante una investigación cualitativa basada en lo social haciendo uso de la narrativa como opción metodológica, la cual -más allá de explorar lo meramente objetivo- se sirva de la palabra para imaginar, representar y dar sentido a la realidad. Esta metodología se preocupa por conocer desde el interior, superando el umbral establecido por datos numéricos o estadísticos y da lugar a un extenso trayecto de investigación. Dicho camino exploratorio quedará delimitado por la voz de alguien que, en el rol de narrador e informante, debe conocer de primera mano aquella realidad que actúe como fundamento para el estudio y compartir generosamente su experiencia. Se trata de la representación subjetiva de un sujeto con base en la cual, contando como pretexto con la perspectiva anónima de quien comparte sus vocablos acerca de la temática abordada, se aspire a alcanzar y hacer más rico el saber.

Palabras claves: Narrativa; Metodología; Experiencia; Palabra; Realidad.

\begin{abstract}
The article presented here aims to describe and publicize the way to proceed with a qualitative research based on the social making use of narrative as a methodological option, which -beyond merely exploring the objective- uses the word to imagine, represent and give meaning to reality. This methodology is concerned with knowing from the inside, exceeding the threshold established by numerical or statistical data. This exploratory path will be delimited by the voice of someone who, in the role of narrator and informant, must know first-hand that reality that acts as a basis for the study and generously share their experience. It's about the subjective representation of a subject on the basis of which, using as a pretext the anonymous perspective of those who share their words about the subject addressed, one aspires to reach and make knowledge richer.
\end{abstract}

Keywords: Narrative; Methodology; Experience; Word; Reality.

Resumo: O artigo aqui apresentado tem como objetivo descrever e dar a conhecer o modo de proceder perante uma pesquisa qualitativa de base social, valendo-se da narrativa como opção metodológica, que -além de explorar o meramente objetivo- utiliza a palavra. imaginar, representar e dar sentido à realidade. Esta metodologia preocupa-se em conhecer de dentro para fora, ultrapassar o limiar estabelecido pelos dados numéricos ou estatísticos e dá origem a um longo percurso de investigação. Este percurso exploratório será delimitado pela voz de quem, no papel de narrador e informante, deve conhecer de perto aquela realidade que serve de base ao estudo e partilhar generosamente a sua experiência. Trata-se da representação subjetiva de um 
sujeito em base à qual, contando como pretexto com a perspectiva anônima de quem compartilha suas vocações sobre a temática abordada, se aspire a alcançar e fazer mais rico o saber.

Palavras-chave: Narrativa; Metodologia; Experiência; Palavra; Realidade.

Cómo citar este artículo: Rueda-Mateos, A. (2020). A favor de la narrativa como metodología para el estudio de lo social. Hachetetepé. Revista científica en Educación y Comunicación, (21), 3-14. https://doi.org/10.25267/Hachetetepe.2020.i21.2

\section{SOBRE LA NARRATIVA MANERA DE PROCEDER}

Parece conveniente destacar desde el inicio la manera en que la narración adquiere un aliciente especial en nuestra época, del mismo modo que lo hiciesen en tiempos pasados quienes inspiraron y dieron forma a relatos sagrados como la Biblia o el Corán. Es en esta tesitura que la investigación narrativa ha ido ganando terreno gradualmente en cuanto a técnica cualitativa para el análisis de datos pues, como refieren Arias y Alvarado (2015), relatar las representaciones mentales y emociones que conforman nuestras vivencias personales ponen de manifiesto una sublime manera de asentar y cohesionar diversos momentos y experiencias.

Así, con esta forma sutil y encantadora de revivir y dar sentido a los hechos, quisiéramos servirnos de una metodología que no se remita únicamente al mero trascender de la cronología y la objetividad, sino que discierna un poco más y emprenda la búsqueda de la razón en cualquier red tanto lógica como arbitraria, intangible y particular. El porqué de este asunto recae sobre cuestiones vinculadas a las continuas transformaciones de tipo social y cultural que se originan a diario y que inquietan nuestro modo de concebir el mundo, el saber e inclusive a nosotros mismos (Sparkes y Devís, 2007).

Frente a dicho cúmulo de ininterrumpidas variaciones y austera perplejidad por el devenir de la era posmoderna, se torna esencial el prestar especial atención al cómo cada cual se nivela, simpatiza y unifica con la realidad que vive, repleta de subjetividad e inseguridad. Se considera por tanto la narrativa como apoyo y favor al entendimiento, la interpretación y la comprensión de unos acontecimientos particulares que se suceden en un ambiente determinado, "además de dotar de significado a aquella parte que estimamos imprescindible: la voz" (Amar, 2018a, p.393).

En esta misma línea, haciendo uso de la narrativa como metodología no numérica y fundamentada en la interpretación, se pretende sacar a relucir la intelección de distintos sucesos relatados por la persona que se presta como informante. Ella, como confidente de ciertas cuestiones introspectivas y con las cuales convive, utilizará su propia palabra para transmitirnos sus pensamientos y reflexiones, su confianza ante las certezas y la suspicacia que deriva de sus dudas, sus argumentos y hasta su mayor o menor (des)motivación. De igual forma que describe García-Huidobro (2016), este punto de vista abre la puerta a redimir aspectos elementales de lo tendencioso o puramente parcial, donde se requiere de múltiples consideraciones para hacer el intento de imaginar la representación que cada sujeto confiere a la realidad en la que se desenvuelve.

Llegados a este punto, inmersos en el desafío narrativo de desvelar lo que encubren los vocablos y el sentir de quien nos informa, poco a poco nos desviaremos de cualquier tendencia relativa a la cantidad mientras nos decantamos por una forma de proceder de condición valorativa. Como enlaza Rivas (2009), los datos serán transferidos del informante al lector -previo paso por el autor- bajo un matiz disímil y sugerente que 
desmonte los modos más tradicionales de observar unos hechos concretos e introducirnos en la investigación. Así, se le otorga un sentido a la realidad que viene dado por la hermenéutica de una subjetividad que aspira a alcanzar y hacer más rico el saber (Bolívar, 2014).

Emplear la narrativa como instrumento para la investigación se acoge a la premisa de descubrir el equilibrio interno de quien habla, encadenando las cualidades de este con el significado aportado a cada uno de los acontecimientos que menciona. Un acto ligado al pensamiento y su parecer, el cual va más allá del examinar lo aparente o superficial. Es ello lo que, de manera muy probable, dé al investigador la capacidad para reparar en las palabras de una forma diferente, organizando el entendimiento y concediéndoles la estructura o formato más acorde a lo que estas tratan de expresar.

En este entramado de nexos perpetuos entre voz y parecer, entre atención y representación, adquiere valor aludir al escaso interés de enmascarar el relato con el que debe contar la persona que nos transmite los hechos, pues a dicho asunto se antepone el aplomo y seriedad concedidos a la narración, así como la madurez de un sujeto, se supone, tan profesional como reflexivo en sí mismo. Este, en la piel de un intérprete que traduce los conceptos internos a la exteriorización de lo acontecido, desarrolla una labor que nos provee de lo complejo de lo real, al abrigo de múltiples opiniones y pensamientos. Aquí, de igual forma que alega Richardson (citado por García-Huidobro, 2016), es tarea del investigador hacer un uso de la narrativa que le ofrezca la posibilidad de exponer, detallar, subrayar y dar visibilidad a determinadas cuestiones con el objeto de ilustrar más y mejor algún aspecto en concreto.

Sencillamente, este artículo tiene la franca intención de preponderar el amplio alcance y sumo provecho contraído por el método narrativo de investigación en cuanto a técnica cualitativa para examinar, considerar e inferir diversos interrogantes vinculados a los datos de un estudio. A ella se le une el valor de la entrevista como instrumento, el cual sirva como pretex to para acceder al conocimiento y, a su vez, brinde a nuestro interlocutor la libertad de manifestar todo cuanto estime y desee.

\section{VIVA VOZ DE SUS PALABRAS}

Situados en el marco de una actuación donde lo cualitativo se apodera de la voz del individuo como fundamento para la investigación, afrontamos la aventura metodológica y ética de revelar declaraciones singulares que actúen como apoyo para descifrar la complejidad de lo social, más allá de estereotipos universales (Márquez et al., 2017). Asimismo, en esta línea de pensamiento donde lo instintivo nos lleva a especular e intervenir, se pretende sondear la influencia que causa en nosotros aquello que oímos $\mathrm{y}$, además, tomar posturas diversas ante la temática y cuestiones que se pretenden investigar. El propósito es claro y viene dado por lo que expresa Rivas (2009), quien lo define con total precisión como el hecho de "comprender mejor la sociedad en que vivimos a partir de la actuación de cada uno y cada una de los que forman parte de ella" (p.29).

Tal inclinación hacia el análisis y el entendimiento de cuanto corresponde a la persona protagonista de nuestra historia, y siempre dentro del escenario en el que se desenvuelve normalmente, hace sopesar el tremendo valer de la narración como técnica para estudios de corte cualitativo dentro del ámbito educativo y social. Narración que implica conocer y conduce al razonamiento, fuente del discurso promovido por preguntas y respuestas que dan forma a la entrevista como momento para llegar al conocimiento

e-ISSN:2172-7910

Doi: 10.25267/Hachetetepe.2020.i21.2

Universidad de Cádiz 
(Kvale, 2011) a través del diálogo. Es dicho diálogo el que, siguiendo a Freire (citado por Verdeja, 2018), "refiere al encuentro que solidariza la reflexión y la acción de sus sujetos orientado a transformar la realidad" (p.49). Pues la palabra, en el departir de investigador e informante, es la piedra angular sobre la que se sustenta el diseño metodológico por el que se opta. De tal modo, reparando en lo mencionado por Vallés (2007), sería posible decir que, gracias al esculpir de la voz de quien nos habla, vamos construyendo una ingeniosa obra maestra -llamada conversación- que compone la trama conceptual y práctica para nuestra labor de indagación.

Profundizando un poco más en el coloquio antedicho, resulta apropiado apuntar que, ocasionalmente, ha de servirse de preguntas de carácter cerrado. Por el contrario, estas deberían tender a la articulación y extensión desmedidas, en una dinámica donde la tertulia permita compartir y contrastar ya no solo palabras, sino también impresiones, sentimientos, ideas y opiniones que subyacen a todo cuanto sea dicho. De semejante manera, dada las circunstancias en la que nos encontramos actualmente, cabría señalar las trabas presentes en el desarrollo de una entrevista presencial, de tú a tú. No obstante, siguiendo las recomendaciones sanitarias establecidas y adaptando las reuniones al valioso contexto virtual del que disponemos, sería posible hacer uso de un extraordinario dispensario de herramientas digitales, las cuales sirvan para reducir las distancias en períodos de confinamiento y faciliten la comunicación de múltiples maneras, como Whatsapp para conversaciones breves de modo rápido y directo, Google Drive para archivar o Google Meet en el caso de reuniones/videoconferencias (Otero et al., 2020).

\section{DE QUIÉN INFORMA Y SU MODO DE INFORMAR}

Decir de la persona escogida para dar vida a nuestro trabajo, a la cual nos referiremos como Niamh a modo de ejemplo para el desarrollo del presente epígrafe, que podría tratarse de alguien rebosante de energía, emotiva al mismo tiempo y de amplia cultura y refinamiento. Por qué no, ella podría ser maestra en ejercicio de Educación Infantil y Primaria, así como Jefa de Estudios en un centro de enseñanza e invertir su escaso tiempo libre en labores de madre de familia numerosa. Supongamos que es extrovertida, sociable, natural y espontánea en la comunicación, fiel a sus principios y que anda convencida de la posibilidad de cambiar y mejorar la educación actual. Consideremos también su virtud para conversar y debatir sobre la temática seleccionada como favor a sus intereses, que podrían no encontrarse alejados de las relaciones sociales, todo lo vinculado con la maternidad y, por supuesto, la educación. Refrendando lo aludido por Patton (1990), de esta manera nos sería posible afirmar que, sin lugar a dudas, hablamos de quien se postula como informante más que acertada, dado su talante, su idoneidad y su razón.

En cuanto al sobrenombre utilizado para mantener el anonimato de nuestro protagonista, apuntar que contamos con múltiples fuentes de las cuales es posible servirnos. Entre otras, en este caso se opta por uno que proviene del gaélico irlandés y, en este mismo idioma, adquiere el significado de "brillante". Dicen que, en la mitología irlandesa, Niamh era la hija de Manannan, dios del mar. A ella, en consonancia con ciertos rasgos que -suponemos- son característicos de nuestra informante, se le atribuye una personalidad jovial, divertida y siempre vanguardista. Además, en ella sobresale el gusto por el disfrute de los avances en cualquier sector -llámese aquí educativo-mientras saca su mejor lado apartando las barreras que encuentra a su paso. 
Tras mostrar algunos aspectos representativos de quien podría tender la mano al ejercicio de articular palabras que nos sirvan como sustento para el análisis, parece oportuno seguir recalcando los motivos que nos llevan a elegir la narrativa como enfoque metodológico a través del cual dar forma al estudio. Entre ellos, sobresale la cabida de tal perspectiva para adentrarse en lo propio o privativo, lo cual, a su vez, pasa del particular sentimiento de pertenencia al disfrute simultáneo de la información. Tanto es así que, de igual forma, dicho modo de proceder serviría para poner de relieve ciertos detalles de una labor diaria que se vincule con la temática abordada, posibilitando que sea percibido ese saber genuino que cada cual constituye a partir de la experiencia (Landín y Sánchez, 2019).

A este tenor, bajo la asidua voluntad de registrar lo que es contado y, al mismo tiempo, la personal forma que tenga nuestro informante de hacérnoslo llegar, debería tratarse de recapacitar y relatar acerca de sus vivencias. Todo lo cual asista a nuestro trabajo desde una doble dimensión -sincrónica (período concreto) y diacrónica (progreso y evolución) - y lo dote de una trascendencia lo más sensata, premeditada y avanzada posible de cara al futuro. Como reseña Amar (2018b):

No pretendemos demostrar, nos basta con mostrar, sabiendo que el diálogo y la escucha activa lo invaden todo, dotando a la acción del beneficio que se apodera del proceso, donde se rompe la dirección jerárquica por otra más participativa inspirada en la entrevista. (p.162)

Es por ello que, en un intervalo de co-elaboración del discurso, se han de seguir patrones propios de la ya señalada escucha activa y mantener un guion de entrevista (semiestructurada), con la intención de recopilar diversas cuestiones vinculadas a la temática investigada, opiniones, etc. El hecho de que el sujeto entrevistado actúe como narrador sienta sus bases sobre la idea de situarnos frente a él y aprovechar su voz como excusa para atender a una historia que, probablemente, merezca la pena que sea oída. Es decir, sus frases serán mucho más que la respuesta a los interrogantes que se le plantean, dado que no sería conveniente que existiera un trayecto claro y delimitado, sino que se persiga el propósito investigativo de acercarnos a nuestro informante para analizar y conocer su compleja realidad, en función de sus relatos y la observación desarrollada durante el transcurso de la investigación.

De este delicado modo sería posible aproximarse a la persona e introducirla de lleno en un estudio de corte narrativo que tome como materia de inspiración asuntos relativos a la seguridad y el compromiso con lo que se hace. Aquí, tras los diversos momentos dedicados al encuentro donde se traten contenidos relativos a lo que se pretende examinar, interpretar y comprender, la declaración de determinadas verdades conllevarán el empleo de la razón y el sentido común como paso previo al uso compartido de estas con los demás para que, posteriormente, puedan ser examinadas. Una faena tan meticulosa como comprometida, la cual entraña un alto grado de compromiso con quien nos habla, de decencia e integridad con todo cuanto resulte del análisis, así como de esmero con los argumentos que se puedan extraer del mismo.

Por último, podría calificarse como esencial la confianza que convendría fuese forjada entre quienes participan de la labor investigativa y la propia tarea de indagación en sí misma, lo cual se preste al hecho de facilitar la planificación, la interacción y la relación de ambas partes (Taylor y Bogdan, 2002). De semejante manera, lo que aquí se menciona también ha de ayudar a revelar ciertos pensamientos e intereses de investigador e informante, culminando todo ello con el intercambio recíproco de ideas y motivando a 
la creación de una intervención afectiva en la búsqueda de una posible mejora en algún ámbito.

\section{DEL INSTRUMENTO AL DETALLE}

Siguiendo a Chase (2015), el instrumento -al que hemos atribuido el común seudónimo de entrevista y que acompaña el calificativo de semiestructurada- debería procurar acceder a la información, primeramente, valiéndose del previo trato y experiencia vivida del investigador junto a su informante. Una herramienta flexible que parta de cuestiones ya preparadas, las cuales cuenten con la posibilidad de ajustarse a nuestro interlocutor con la intención de acrecentar su grado de motivación, aclarar y precisar términos e ideas, así como reconocer posibles ambigüedades o disminuir los formalismos. Del mismo modo que señalan Abarca et al., (2013), este proceso de recolección se sustenta sobre las bases que quedan establecidas por el intercambio comunicativo llevado a cabo por dos -o más- personas. Dicha técnica requiere de ciertas condiciones que resulten ser apropiadas y favorables para el acto de conversación, lo cual invite a nuestro informante a compartir sus experiencias de una manera abierta y cómoda.

Más adelante, en cada sesión sería posible disponer de un modelo abierto para llevar a cabo el estudio, basado en una serie de cuestiones de investigación poco restringidas y lejos de respuestas únicas o predecibles, sin desaprovechar la oportunidad de introducir preguntas espontáneas para completar la información. Estos, repletos de cuestiones que toman la forma de preguntas abiertas y no dicotómicas, propias de estudios cualitativos (Kvale, 2011), cuentan con la posibilidad de ser facilitados a nuestro interlocutor en el momento previsto para cada una de las reuniones.

Mientras la recogida de datos tenga lugar a través de las anotaciones oportunas por parte del investigador en el cuaderno de campo, las distintas conversaciones podrían ser grabadas en archivo de audio para que posteriormente puedan ser transcritas a texto, además de analizadas. Todo ello, preservando siempre la utilidad o conveniencia del tópico indagado, se ve configurado por las palabras de nuestro informante como molde que aporta sentido al método seleccionado y, en general, a la temática que nos concierne. Una tarea desprovista de interpretación pero con cierto grado intencionado de ambigüedad, en la cual el entrevistado imitará el papel propio de quien narra un cuento, mientras el investigador -o quien formula las preguntas- pasará a ser mero asistente a un detallado discurso, útil para su posterior y consecuente lectura en profundidad.

Parece conveniente destacar también que la narrativa, como esencia del método aquí descrito, se convierte en una de las intenciones principales que podrían perseguirse con el trabajo a desarrollar, siendo su integridad la que le confiera cierta "consistencia y fiabilidad de cara a su validez científica" (Delgado-García, 2018, p.7). En cuanto al procedimiento a seguir para dotar de validez a las entrevistas puede decirse que, en un primer momento y tras ser diseñadas, sería interesante que fuesen valoradas por un grupo de expertos, los cuales lleven a cabo un juicio crítico a través de ciertas aportaciones encaminadas a la mejora de las mismas (McMillan y Schumacher, 2005). Son estos especialistas quienes, contando con formación en la materia en cuestión, puede decirse que conjuntamente gozan de las características esenciales que los posicionan como técnicos dentro de los campos sobre los cuales se alce el estudio. Luego, los distintos comentarios han de ser sopesados y, por último, efectuarse una remodelación general de todo el guion con base en los aportes realizados. 
Por otra parte, ante un universo narrativo que incita a la interpretación, las estrategias para afrontar el difícil quehacer de analizar y representar lo narrado necesitarían contar con el apoyo del escaso interés por cooperar con variables de esta índole, en ocasiones inherentes a las circunstancias o propias de la mente humana, lo cual las hace más lícitas y honestas en sí mismas. Guiados unas veces por captar y otras por expresar al pie de la letra - $\mathrm{o}$ de la voz en nuestro caso- el parecer de quien informa, al mismo tiempo que regidos por el respeto del lenguaje y el contexto referidos, se insiste en el intento de revivir, rememorar y encadenar sus palabras bajo el criterio de la reflexión. Pero, en cualquier caso, lo que prevalece es la intención de concebir y compartir los hechos que nos competen (Rivas, 2009), convirtiendo lo abstracto en entendible o encontrando lo impreciso valores concretos.

Dicho de otro modo, este pensamiento podría quedar expresado, al igual que refiere Amar (2020), de la siguiente manera:

La entrevista en la investigación cualitativa-narrativa se reviste de comprensión, más que en buscar categorías. Y, es por ello, que mantendremos la tendencia a la búsqueda de agrupaciones temáticas, [...] así como iremos a destacar su pertinente análisis de contenidos y la extracción de consideraciones inspiradas en la atención y en la madurez, alejándonos de los apriorismos y la precipitación por llegar a un final o a unas conclusiones generales. (p.116)

Siguiendo a este mismo autor, resulta útil mencionar que los datos podrían ser organizados según la disposición establecida por los distintos temas que el informante expone en su departir, adquiriendo un papel fundamental el compromiso ético del que se dispone como matiz que determina la tarea de indagación (Wolff-Michael y Hella, 2018 citados por Amar, 2020). Indistintamente, un sinfín de conversaciones exentas de límites espacio-temporales, a nivel formal e informal, que contribuyan a elaborar el trabajo de investigación pretendido y moldear una relación más estrecha, cercana y sincera entre quien investiga y es "investigado".

En consonancia con todo esto, el conjunto de acciones desarrolladas deberían tratar de poner en juego tanto la voz interna como externa de quien nos habla en la búsqueda de un problema, una solución o una explicación. Es decir, teniendo muy presente el principio investigativo del que parta el estudio y siguiendo a Moriña (2017), una serie de actuaciones libres de prejuicios encaminadas al conocimiento y comprensión de la realidad concreta de la informante, para lo cual nos valemos de su propia narrativa.

\section{LA PLENITUD DE UN TRAYECTO (IN)FORMATIVO}

En cuanto al fin pretendido con este apartado de naturaleza metodológica entendiendo por metodología la manera en que, a menudo, se hace frente a las dificultades y se emprende la búsqueda de posibles soluciones, o también según Rist (citado por Taylor y Bodgan, 2002) el "modo de encarar el mundo empírico" (p.20)-, cabría destacar que se acerca bastante al hecho de argumentar y entrar en detalles en lo referido a los pilares sobres los cuales necesitaría sostenerse la labor de recogida de datos. Una labor que precisaría del empleo de diversas fuentes, herramientas y procedimientos para ser efectuada. Todo ello sobre el colchón de ciertas cuestiones como el qué y cómo se ha llevado a cabo, además de otras como el por qué es lo que mejor se adapta a la situación y, no menos importante, resulta ser afín y estar sujeto con el concepto de indagación en torno al cual gire nuestro estudio. 
Otra cuestión interesante aquí es que, tratándose de un estudio de corte cualitativo, sería conveniente mirar por el cuidado de los distintos aspectos ligados al ámbito de lo moral. Dicho de otra manera, en el intento de aproximación al marco social en el que se centre la problemática, deberían seleccionarse diversos principios éticos -negociación, colaboración, confidencialidad, imparcialidad y equidad (Vázquez y Angulo, 2003) - que justifiquen el compromiso existente entre la labor de indagación a llevar a cabo, la temática en cuestión y la sociedad, con el fin de dar forma a un conocimiento plausible, creíble y legítimo. Así, este asunto podría verse favorecido con el respaldo de una serie de criterios que proponen autores como Angulo (1993), Kemmis y Robottom (1981) o Simons (1987 y 1989):

\section{Tabla 1}

Principios y criterios éticos

\begin{tabular}{|c|c|}
\hline Principio & Criterios y características éticas \\
\hline Negociación & $\begin{array}{l}\text { A partir del acceso al contexto y la } \\
\text { información hasta llegar al valor de esta, } \\
\text { así como el de las conclusiones. }\end{array}$ \\
\hline Colaboración & $\begin{array}{l}\text { Abarcando la participación de la persona } \\
\text { informante. }\end{array}$ \\
\hline Confidencialidad & $\begin{array}{l}\text { Dando en todo momento un papel } \\
\text { principal al respeto por la condición } \\
\text { anónima de la información recogida y los } \\
\text { procesos estipulados. }\end{array}$ \\
\hline Imparcialidad & $\begin{array}{l}\text { Venerando diversas representaciones y } \\
\text { opiniones sobre la temática abordada. }\end{array}$ \\
\hline Equidad & $\begin{array}{l}\text { Haciendo uso de la búsqueda de } \\
\text { información sola y exclusivamente como } \\
\text { base potencial de cara a un proceso de } \\
\text { mejora que no perjudique ni afecte de } \\
\text { manera negativa a ningún particular o } \\
\text { colectivo, preservando caminos para la } \\
\text { objeción y el debate de las conclusiones } \\
\text { resultantes. }\end{array}$ \\
\hline Compromiso con el conocimiento & $\begin{array}{l}\text { Priorizando el papel de la responsabilidad, } \\
\text { tanto individual como colectiva de la } \\
\text { investigación en sí, pretendiendo } \\
\text { visibilizar ciertas causas y fundamentos } \\
\text { que constituyen los acontecimientos y } \\
\text { motivos para la indagación. }\end{array}$ \\
\hline
\end{tabular}

Fuente. Elaboración propia

En otro orden de ideas, la selección del caso y el informante requiere estar sujeta a la elaboración del saber desde una óptica sensata, consciente, intencionada y sistemática de la realidad estudiada. Dicho asunto, asentado sobre la riqueza de la información y la mayor o menor facilidad para el acceso a ella, servirá como soporte a otras cuestiones 
previas relacionadas con los pareceres e impresiones derivados de la observación y contribuirá a que el estudio se nutra de progresiva confianza, seguridad y profundidad en el transcurso del período dedicado al mismo.

De esta manera, en virtud de razones meramente prácticas -supongamos que se dispone de insuficiente y limitado plazo de tiempo disponible o que se encuentran ciertas complicaciones en la aplicación-, la tarea de investigación podría quedar establecida sobre la labor de observación, la revisión bibliográfica y documental, un diario de campo, así como la mediación a través de los ya mencionados momentos de entrevista, los cuales contribuyan a la obtención de otra versión a favor de una heterogeneidad y mayor aseveración de los datos empíricos que anteriormente puedan haber sido reunidos. Un propósito orientado a recopilar información real que aspire a atender, conocer y comprender la realidad de nuestro informante, enfocándolo en un contexto y una situación específica.

En consonancia con todo esto, partiendo desde la realidad particular seleccionada y teniendo como eje central el propósito general de la investigación, deberían alzarse una serie de preguntas que adquieran el papel de guía para la recuperación de la información, su análisis, el observar e ir más allá, además de contribuir al entendimiento e interpretación de los motivos que provocan que algo sea de una determinada manera y no de otra.

\section{CORTOS PASOS SOBRE UN LARGO CAMINO}

Bajo el pretexto de lograr un acercamiento a la realidad seleccionada, y como se viene mencionando a lo largo de todo este artículo, se ha de utilizar el método narrativo para recoger ciertos relatos de vivencias personales del sujeto que nos informa. Una aproximación de tipo social en la que, al igual que apunta Bolívar (2002), el protagonista tome como punto de partida su propia experiencia particular y, sirviéndose de la reflexión, lleve a cabo una reconstrucción única y original de la misma, siendo esta última la empleada por quien investiga para reflejar lo acontecido.

En este sentido, se abrirá la veda de un extenso camino orientado a lo individual, lo privativo de quien nos habla y el exclusivo modo que él tiene de hacerlo. Una senda plagada de pasos exploratorios y registros incesantes acerca de todo aquello que es mencionado y podría tener valía o ser de interés para el estudio, garantizando la delimitación y la singularidad de los sucesos más elocuentes (Flick, 2007). Es así que, contando con esfuerzos centrados en el descubrimiento de percepciones, será posible dar forma a un relato reflexivo y generoso, cualidades básicas de la labor a desarrollar y que, como parte fundamental del análisis de la realidad intrapersonal e interpersonal del informante, se originarán en él y llegarán al lector, previo paso por el autor -o investigador en nuestro caso-. Y, seguramente, todo ello con el propósito de compartir sentimientos, pensamientos, experiencias, saberes, etc., en el intento de prosperar como personas y, no menos importante, mejorar aquello sobre lo cual estemos investigando. De manera más concreta, viene a definirse una tarea de indagación constituida en favor de la confrontación y la solidez de una versión inicial que persiga el fin de producir variedad y obtener argumentos razonados gracias a la generosidad del informante.

Como señalan Martín-Alonso et al. (2019), una conversación que sea suscitada por el deseo de búsqueda, interpretación y construcción de un sentido acerca de la experiencia vivida y que esté forjada sobre la base de una serie de cuestiones previamente establecidas pero, análogamente, abiertas a la improvisación. Estas, como senderos que conducen al 
camino establecido por una secuencia vocal que suene durante cada sesión de entrevista, evidenciarán un aporte simultáneo y participativo en lo que al saber, reflexión y aprendizaje bilateral del hecho narrativo se refiere, y actuarán como complemento inmejorable a la revisión bibliográfica y documental que haya sido efectuada con anterioridad.

Finalmente, como colofón a un recorrido desigual, a una andadura sensitiva o a una ruta plagada de inventiva -llámenlo como prefieran-, de esta manera habremos logrado recopilar un conocimiento dialogado y orientado a la búsqueda de miradas, sonrisas, gestos, sueños, entre otras cuestiones estrechamente ligadas a la temática sobre la que estemos trabajando. Se torna interesante resaltar cómo, guiados sobre una "nube" de palabras, a lo largo de las distintas fases que conforman el proceso deberían sucederse múltiples situaciones y encuentros en los que predomine la comunicación y la colaboración con el informante, lo cual haga posible ahondar un poco más en la realidad investigada.

Y es que el conversar con nuestro interlocutor tendría que servirnos como favor al análisis e interpretación de una serie de datos que contribuyan a dar respuesta a las metas del estudio previamente establecidas y arrojar ciertas conclusiones al respecto. Porque él protagonizará la historia en la que se base nuestra investigación narrativa y de la cual nos sea posible aprender de y con alguien realmente comprometido con su labor. Largo y bonito pasaje que podría tener así su punto de partida y que, desde tal momento, ha de ser emprendido con la satisfacción y el impulso de pensamientos como qué mejor que empezar para llegar a cambiar. Un cambio que, a su vez, podría verse apoyado y profundizado por novedosas e incipientes líneas de indagación que traten acerca de diversas temáticas y que sean el complemento idóneo a la puerta que, con nuestro trabajo, hayamos abierto al saber.

Estas no son más que las notas finales de los acordes que componen la melodía de una opción metodológica considerada más que apropiada para investigaciones con carácter socioeducativo. Estudios de indagación en y para la enseñanza en los que la consigna ha de ser simple: hay que conocer para poder comprender. Y ello es lo que se quisiera transmitir con estas páginas, como intento de comprensión e interpretación que mira al futuro personal y colectivo con las gafas de la corresponsabilidad. Un futuro, hoy incierto, pero que esperamos cuente con alicientes suficientes para redimensionar, transformar y mejorar la realidad del presente. Un futuro que queda lejos, pero cada vez está más cerca y sobre el cual, juntos, hemos de tomar buenas decisiones y actuar para superar cada resquicio que nos conciba como desiguales. Un futuro en el que, confiemos, todo saldrá bien.

\section{REFERENCIAS BIBLIOGRÁFICAS}

Abarca, A., Alpízar, F., Sibaja, G., y Rojas, C. (2013). Técnicas cualitativas de investigación. Granada: Editorial UCR.

Amar, V. (2018a). Déjame que mire un cuento: Narración, familia y educación infantil. Una investigación narrativa. Profesorado: Revista de currículum y formación del profesorado, 22(2), 389-405. https://doi.org/10.30827/profesorado.v22i2.7729

Amar, V. (2018b). Miradas y voces de futuros maestros. Barcelona: Octaedro.

Amar, V. (2020). Un camino para la educación para la paz: Una investigación narrativa.

$\begin{array}{llllll}\text { Revista de Paz y Conflictos, } & 13(1), & 111-125 . \\ \text { http://dx.doi.org/10.30827/revpaz.v13i1.11362 }\end{array}$

e-ISSN:2172-7910

Doi: 10.25267/Hachetetepe.2020.i21.2

Universidad de Cádiz 
Angulo, J. (1993). La evaluación del proyecto curricular de centro o cómo ampliar la autonomía profesional del docente en tiempos de burocracia flexible. Kikiriki, (30), 41-48.

Arias, A. M. y Alvarado, S. V. (2015). Investigación narrativa: apuesta metodológica para la construcción social de conocimientos científicos. Revista CES Psicología, 8(2), 171-181.

Bolívar, A. (2002). “¿De nobis ipsis silemus?”: Epistemología de la investigación biográfico-narrativa en educación. Revista Electrónica de Investigación Educativa, 4(1). Disponible en: http://redie.uabc.uabc.mx/vol4no1/contenidobolivar.htm

Bolívar, A. (2014). Las historias de vida del profesorado. Voces y contextos. Revista Mexicana de Investigación Educativa, 62(19), 711-734.

Chase, S. (2015). Investigación narrativa: Multiplicidad de enfoques, perspectivas y voces. En I. Denzin y N. Licoln (Coords.), IV Manual de Investigación Cualitativa: Métodos de recolección y análisis de datos (pp. 58-112). Barcelona: Gedisa.

Delgado-García, M. (2018). La metodología biográfica narrativa y posibilidades en el ámbito del docente universitario. Revista Latinoamericana de Metodología de Las Ciencias Sociales, 8(2), e043 .https://doi.org/10.24215/18537863e043

Flick, U. (2007). Introducción a la investigación cualitativa. Madrid: Morata.

García-Huidobro, R. (2016). La narrativa como método desencadenante y producción teórica en la investigación cualitativa. EMPIRIA. Revista de Metodología de las Ciencias Sociales, (34), 155-177. https://doi.org/10.5944/empiria.34.2016.16526

Kemmis, S. y Robottom, I. (1981). Principles of procedure in curriculum evaluation. Journal of Curriculum Studies, 13(2), 151-155.

Kvale, S. (2011). Las entrevistas en investigación cualitativa. Madrid:Morata.

Landín, M. del R. y Sánchez, S. I. (2019). El método biográfico-narrativo: una herramienta para la investigación educativa. Educación, 28(54), 227-242. https://doi.org/10.18800/educacion.201901.011

Márquez, M. J., Prados, M. E. y Padua, D. (2017). La metodología biográfica narrativa en la formación del profesorado. En R. Zapata, R. Dalouh, V. Cala y A. González (Eds.), Educación, salud y TIC en contextos multiculturales: Nuevos espacios de intervención (pp. 326-336). Almeria: Universidad de Almería.

Martín-Alonso, D., Blanco, N. y Sierra, J. E. (2019). Comprensión pe-dagógica y construcción de la relación educativa. Una indagación narrativa. Teoría de la educación, 31(1), 103-122. https://doi.org/10.14201/teri.19442

McMillan, J. H. y Schumacher, S. (2005). Investigación educativa. Madrid: Pearson.

Moriña, A. (2017). Investigar con historias de vida. Metodología biográfico narrativa. Madrid: Narcea.

Otero, L., Díaz, M. I. y Pandiella, R. (2020). Herramientas digitales para la comunicación, la tele-docencia y la tele-orientación educativa en tiempos de COVID-19. Revista AOSMA, (28), 92-103.

Patton, M. Q. (1990). Qualitative evaluation and research methods.Newbury Park: Sage.

Rivas, J. I. (2009). Narración, conocimiento y realidad. Un cambio de argumento en la investigación educativa. En J. I. Rivas y D. Herrera (Eds.), Voz y educación. La narrativa como enfoque de interpretación de la realidad (pp. 17-36). Barcelona: Octaedro. 
Simons, H. (1987). Getting to Know Schools in a Democracy. The Politics and Process of Evaluation. The Falmer Press.

Simons, H. (1989). Ethics of Case Study in Educational Research and Evaluation. En R. Burgess (Ed.), The ethics of educational research (pp. 114-138). Gran Bretaña: The Falmer Press.

Sparkes, A. y Devís, J. (2007). Investigación narrativa y sus formas de análisis: una visión desde la educación física y el deporte. En W. Moreno y S.M. Pulido (Eds.), Educación, cuerpo y ciudad: el cuerpo en las interacciones e instituciones sociales (43-68). Medelín: Funámbulos.

Taylor, S. y Bogdan, R. (2002). Introducción a los métodos cualitativos de investigación: la búsqueda de significados. Barcelona: Paidós.

Vallés, M. (2007). Entrevistas cualitativas. Centro de investigaciones sociológicas.

Vázquez, R. y Angulo, J. F. (2003). Introducción a los estudios de casos. Los primeros contactos con la investigación etnográfica. Málaga: Aljibe.

Verdeja, M. (2018). Ideas centrales del pensamiento pedagógico político de Paulo Freire: dimensiones de análisis. Revista Fuentes, 20(1), 43-56. https://doi.org/10.12795/revistafuentes.2018.v20.i1.03 